\title{
Exploration of System Performance of Quantum Cryptography in Network Security
}

\author{
Velpula Sundara Ratnam
}

\begin{abstract}
Cryptography NetworkSecurity was a conception to secure the datatransmission and the network of the wireless networks. For the datatransmission the major feature isdata Security on independent network. In a network authorization of access of thedata was maintained by the NetworkSecurity, here the network administrator controls the entire process. The users will login by the ID, Password and some other authenticating information to access in to the network to perform their authentication. The NetworkSecurity protects different kinds of both private and public networks, computer networks and daily transactions in the business and in the communications and in government and other sectors. Public access and private access are the different varieties of Networks in the system. In this paper the cryptography NetworkSecurity by Ciphers are defined. In this cryptography algorithm is used.
\end{abstract}

\section{INTRODUCTION}

$\mathrm{I}_{\mathrm{n}}$ In the information security the most important is NetworkSecurity, because it protects all the information which is transferring through all the networks. NetworkSecurity defines to all the software and hardware functions, operationalprocedures, features, measures,algorithms, access control, administrative policy and the management policy obligatory to access the adequate level of security for the software and hardware, and thedata information in the network. NetworkSecurity problems are differentiate into four closely interweaved areas: authentication, integrity control, nonrepudiation and security. Privacy is also known as Secrecy had to keep the information securely from the out of hands of the non-authorizers. In the networks authentication compacts with the decisive whom you are sharing your words and transactions and before enlightening sensitive information about a business deal. With signatures the Nonrepudiation deals are done. Data Integrity: If the sender and the receiver is accomplished to authenticate and their conversation also need to secure that the conversation of their communication is not changed, either by maliciously in the transmission. Check summing methods that come across in thedata link protocols and reliable transport of thedata was extended. The emerging technology in the NetworkSecurity is Cryptography. An Extensive utilization of the computerizedata storage, transmission and processing gives sensitives. Personal and securedata information susceptible to non-authorized access while in transmission or storage. Due to drastic updating in the communication networks and spying technologies, private networks and business organizations are beginning to secure theirdata in the computers and in the networks utilizing cryptography techniques, which were newly updated in the diplomatic and military communications. In the today's worldcryptography is the most vital technique in the

Revised Manuscript Received on December 22, 2019.

VELPULA SUNDARA RATNAM, Associate Professor In Cse Department, Mallareddy engineering college for women (autonomous institution-ugc,govt of india) maisammaguda, secunderabad-100 computers and in the NetworkSecurity. Securing in the bank transactions, communication networks, internet shopping and business mails. By using the traditional and the updatedcryptography different mathematical methods are neglected eavesdroppers by copying the conversations of the encrypted data. Networks and computers which are using for thedata storage and for the communicating and processing of thedata are secure by the non-authorized accesses.

There is an encryption in the general transferring and storage of thedata over communication networks which are insecure by the normal transferring and storage, for this in this paper Quantumcryptography is proposed. To secure the encryptionkeyby the perfect security the Quantumcryptography was used to exchange them. Julius Caesar proposed the normal cryptography[7] to secure the knowing of his data. Caesar cipher used mono-alphabetic cryptosystem[7] to secure the same texted alphabet of the original one.

Moreover the methods of the receiver and the source, here the channel codes are updated notations in the information theory they have the roots. In 1948 Claude Shannon was proposed the information theory for the secrecy basis. That defines the uncertainty can be proposed into the encodeddata will not be more than that the cryptographickeyutilized for encoding this[9]. In 1949, Claude Shannon proposed this method for the security communication, i.e., that the encryption method is absolutely secure, if there are any twodatasets $D_{1}$ and $D_{2}$, and cipherind $C$ had as it is prospect into existence this encryptions of the $D_{1}$ was starting of the encryption of $\mathrm{D}_{2}$ [6]. Shannon also proposed two minor cryptographic methods: confusion \& diffused. Among the authors confusions method meaning into anywhere other technique in that varieties statistically relationships into betweenkeythe ciphertext as problematic as possible. Diffusion was the normal term to encryption method that expanded in the statistical properties of thekeyand plain text over a variety into bit of cipher texts.

\section{CRYTOGRAPHIC PRINCIPLES}

Redundancy Cryptographic principle 1:

Redundancy is the first principle to encrypt the data, i.e. information doesn't required to understand the data. Data should contains at least some redundancy.

Freshness Cryptographic principle 2:

For the Foil replay attacks required some other techniques. One of the method is counting the delay time of thedata and it holds only for $10 \mathrm{sec}$. at the receiver just hold thedata for 10 sec, to compile with freshly updated data. This will be rejected if it holds more than 10 sec. 


\section{Exploration of System Performance of Quantum Cryptography in Network Security}

\section{CRYPTOSYSTEM TYPES}

In over-allof the cryptosystems are classifications into 2 types symmetric and the asymmetric. Contingent only on eithen. keys at a receiver and transmitter are computed easily from one another. Differentkey in asymmetriccryptography is utilized for decryption and encryption. Bob and Alicecan share the samekey $(\mathrm{K})$ of symmetric encryption is unknown to attacker. Utilizes to decrypt and encrypt of them communication network.

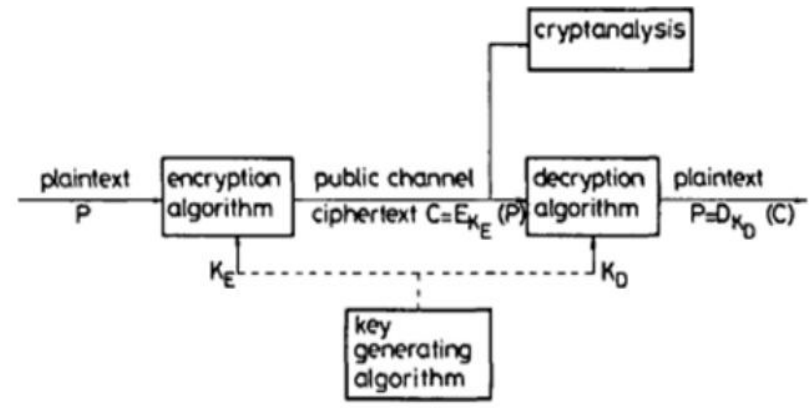

Fig. 1 The Generally as secured into system

Cryptographicsystems is utilized into stretch security \& attestation in communication systems and in PC. As appeared into Figure.1, encryptionalgorithms encipher on plain texts, \& clear information, into twisted ciphertext \& cryptogram utilizes as the keys. The decipher estimation is utilized for unscrambling or decipher so as reestablish a primary data. Cryptosystem is towards as symmetrically, into while it is cases in together an encipher \& decipher key must be stayed thoughtful, as asymmetrically, to when it is each one as a key is to open except trading off another. A. Asymmetriccryptosystems. They have been reasonable issues related for an age, development $\&$ assurance at the enormous numbers into key. The reaction for them key distributions issue has proposed in 1976 between Hellman \& Diffie [10]. The sort of figure was proposed which uses two one of the sortkeys: 1key utilized with encipher may make open, which is other, utilized with decipher, as keep on riddle. The 2 key is made to liked the degree, (i.e), as computational as infeasible into discovered as a conundrumkey from general society key. On the off chance that client the necessities to chat with client $\mathrm{B}$, the can use B's openkey (from an open library) to encipher an information. No one anyway B can translate a ciphertext since just he has a mystery deciphering key. A course of action depicted above is known as an openkey cryptosystem or an asymmetric cryptosystem[11]. On the off chance that asymmetricalgorithms fulfill certain limitations, they can in like way be utilized for making insisted modernized signatures[12]. B. Symmetric cryptosystems in symmetric cryptosystems (comparably called standard, mysterykey or one-key cryptosystems), an enciphering and deciphering keys are either vague or essentially related. One of them can be suitably gotten from other. The 2 keys must be kept quiet, and if either is undermined further secure communication is incomprehensible. Keys should be traded between clients, routinely over the moderate secure channel, for instance the private emissary, and a measure of keys can be enormous, if each pair of clients requires the substitute key, in any event, for the moderate number of clients, for example $n(n-1) / 2$ for $\mathrm{n}$ clients. This makes the key-dispersal issue which is somewhat unraveled in an asymmetric systems. Instances of symmetric systems are an information encryption standard (DES) [4] and rotor figures.

\section{CRYPTOGRAPHIC MODELS AND ALGORITHM}

\section{Encryption model}

They are 2encryption models, and it is described below: Symmetricencryption and Asymmetricencryption. In Symmetricencryption, Encryptionkey = Decryption key. In Asymmetricencryption, Encryptionkey ${ }^{1}$ Decryption key

\section{Publickey Cryptography}

It is otherwise called Asymmetric Scheme since you are utilizing two keys Public and Private

At cryptographicframework has been utilizations 2 key is to openkey called as to everybody \& private or mysterykey called as distinctly as the benefits into the informations. These are pointing into which is John requires as sending into an protects as informations into Jane, he utilizes Jane's openkey as run the informations. Jane than used to him privatekey to unscramble it.

At significant components as the generally populationkeyframework to the normal population \& privatekeys is connects in so that lone the general populationkeycan is utilizes as run the information's \& now the comparing privatekey may be utilizes as decodes in their. The additionally, In the terms of $\&$ purposing model difficultly to finding then the privatekey into events of has been known as people into general keys.

Openkey systems, in this examples, super Good Privacy (PGP), is turning out to be famous for transmittingdata through the Internet. They are incredibly secure and generally easy to utilize. The main trouble with openkey systems is that you have to realize the beneficiary's openkey to encode a message for the person in question. What's required, along these lines, is a worldwide vault of open keys, which is one of the guarantees of the new LDAP innovation. Open keycryptography was imagined in 1976 by Whitfield Diffie and Martin Hellman. Therefore, it is at some point called Diffie-Hellman encryption. It is additionally called asymmetric encryption since it utilizes two keys rather than 1 key (symmetric encryption).

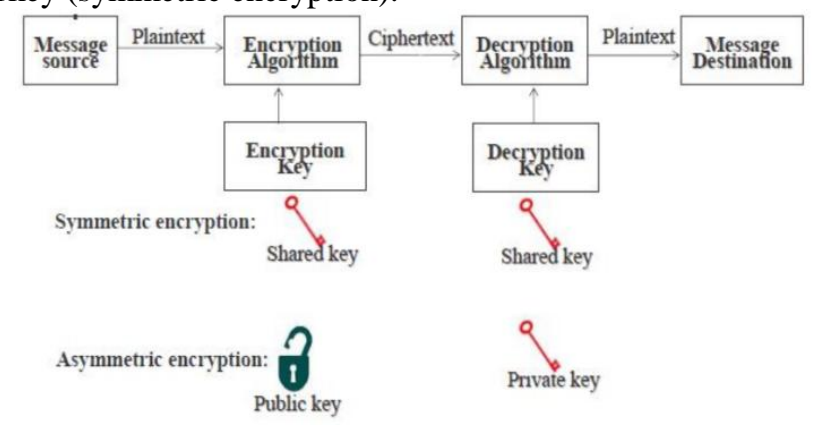

Fig 2: Cryptography. 


\section{B. Algorithm}

Cryptographic wide range algorithms

1) DES: This is the 'Data Encryption Standard'.

2) RSA: RSA is a public-key system.

3) HASH: A 'hash algorithm' is used for computing a shortened depiction of fixed length data/file.

4) MD5: MD5 is a 128 bit message digest function.

5) AES: This is the Advanced Encryption Standard

6) SHA-1: SHA-1 is hash 9lgorithm similar in structure to $\mathrm{MD}$ 5.

\section{CONCLUSION}

NetworkSecurity is the most basic portion in informationsecurity since it is obligated for checking all information experienced networkedcomputers. NetworkSecurity contains the game plans made in a concealed PC network establishment, procedures got through networks executive into guarantee this networks \& networks as open resource with unapproved get to, \& unsurprising \& steady watching \& estimation into sufficiency is need combinations. They have thought about several cryptographies strategies into grow into securityofnetwork. Cryptographic, towards as proper communications shows, may give an elevated level of security in cutting edge communications against intruder ambushes also as the communication between two one of a kind computers is concerned.

\section{REFERENCES}

1. DENNING, D., and DENNING, P.J.: 'Information security', ACM Comput. Reviews, 1979, 11, pp. 227-250 [2] A Role-Based Trusted Network Provides Pervasive Security and Compliance - meet with JayshreeUllal, senior VP of Cisco.

2. Dave Dittrich, Network observing/Intrusion Detection Systems (IDS), University of Washington.

3. 'Information encyption standard', FIPS PUB 46, National Bureau of Standards, Washington, DC Jan. 1977

4. Murat Fiskiran, Ruby B. Lee, - Workload Characterization of Elliptic Curvecryptography and different NetworkSecurityalgorithms for Constrained Environmentsl, IEEE International Workshop on Workload Characterization, 2002. WWC-5. 2002

5. Coron, J. S. , " What is cryptography?", IEEE Security and Privacy Journal, 12(8), 2006, p. 70-73.

6. Pfleeger, C. P., \&Pfleeger, S. L.," Security in Computing", Upper Saddle River, NJ: Prentice Hall.2003.

7. Salomon, D., " Codingfordata and Computer Communications", New York, NY: Spring Science and Business Media. 2005.

8. Shannon, E. C., "Correspondence hypothesis of mystery framework", Bell System Technical Journal, Vol.28, No.4, 1949, pp.656-715.

9. DIFFIE, W., and HELLMAN, M.: 'New bearings in cryptography', IEEE Trans., 1976, IT-22, pp. 644-654

10. SIMMONS, G.J.: 'Symmetric and hilter kilter encryption', ACM Comput. Overviews, 1979, 11, pp. 305-330

11. RIVEST, R.L., SHAMIR, An., and ADLEMAN, L: 'A strategy for getting computerized marks and open key cryptosystems', CACM, 1978,21 , pp. $120-126$ 